\title{
Tributo ao centenário da Síndrome de Waterhouse-Friderichsen: relato de caso
}

\author{
Tribute to the centenary of Waterhouse-Friderichsen syndrome: a case report \\ Huei Hsin Shieha, Patrícia Freitas Góes ${ }^{a}$, Fabiana Roberto Limab, \\ Maria Cláudia Nogueira Zerbinib ${ }^{\text {, Albert Bousso }}{ }^{a}$
}

Shieh HH, Góes PF, Lima FR, Zerbini MCN, Bousso A. Tributo ao centenário da Síndrome de Waterhouse-Friderichsen: relato de caso. Autopsy Case Rep [Internet]. 2011;1(1):42-52.

\section{RESUMO}

A síndrome de Waterhouse-Friderichsen teve o relato pioneiro de Rupert Waterhouse há exatamente 100 anos em 4 de março de 1911. É uma síndrome infecciosa aguda de evolução catastrófica que requer a utilização de todos os recursos disponíveis de terapia intensiva e geralmente evolui para o êxito letal. Nós descrevemos um caso ilustrativo de meningite meningocócica $\mathrm{C}$ que ilustra aspectos clínicos, terapêuticos e anatomopatológicos de uma criança de 3 anos e 4 meses atendida em um Hospital Universitário com evolução fatal, apesar da aderência às recomendações vigentes atualizadas para o tratamento do choque séptico pediátrico. Salientamos a pouca mudança no aspecto clínico e anatomopatológico da síndrome de Waterhouse-Friderichsen nos últimos 100 anos, apesar da enorme evolução terapêutica e tecnológica que acompanha a terapia intensiva pediátrica.

Unitermos: Síndrome de Waterhouse-Friderichsen; Púrpura fulminante; Choque séptico; Meningite meningocócica; Insuficiência Adrenal.

\section{ABSTRACT}

The Waterhouse-Friderichsen Syndrome was first reported by Rupert Waterhouse exactly 100 years ago on March 4th, 1911. It is an acute infectious disease that generally progress to a lethal outcome despite the adoption of all available resources of an intensive therapy unit. We describe an illustrative case of meningococcal meningitis $C$. This report details the clinical, pathological and therapeutic features of a patient of 3 years and 4 months who had a fulminating evolution, despite adherence to updated recommendations for the treatment of pediatric septic shock. We stress the little change in clinical and pathological aspects of Waterhouse-Friderichsen Syndrome during the last 100 years, despite tremendous advances in therapy and technology of the pediatric intensive care.

Keywords: Waterhouse-Friderichsen Syndrome, Purpura fulminans, Shock septic; Meningitis meningococcal; Adrenal insufficiency.

\footnotetext{
${ }^{\text {a} D i v i s a ̃ o ~ d e ~ C l i ́ n i c a ~ P e d i a ́ t r i c a ~-~ H o s p i t a l ~ U n i v e r s i t a ́ r i o ~-~ U n i v e r s i d a d e ~ d e ~ S a ̃ o ~ P a u l o ~-~ S a ̃ o ~ P a u l o / S P ~-~ B r a s i l . ~}$

bServiço de Anatomia Patológica - Hospital Universitário - Universidade de São Paulo - São Paulo/SP - Brasil.
} 


\section{INTRODUÇÃO}

A primeira descrição da síndrome de Waterhouse-Friderichsen (SWF) completa 100 anos $^{1}$. Este centenário remete-nos ao artigo original de 04 de março de 1911 do Dr. Rupert Waterhouse (1873-1958) com um pequeno texto de página e meia no Lancet intitulado A case of suprarenal apoplexy $y^{1,2}$. Dr. Waterhouse fez o relato do caso clínico de um menino de 8 anos de idade documentando o início do quadro febril, o aparecimento de sufusões purpúricas dentro do hospital, a piora clínica evidente e anotou a avaliação médica evolutiva durante os seus cuidados no Royal United Hospital, Bath, Inglaterra, onde era médico assistente e patologista. O paciente morreu após 4 horas e Dr. Waterhouse procedeu à autópsia. ${ }^{1}$

Embora este artigo pioneiro de SWF não tenha sido a primeira descrição sobre apoplexia suprarenal ${ }^{1,2}$, Dr. Waterhouse teve o mérito de descrever detalhadamente o quadro fulminante da SWF como uma nova entidade clínica, incluindo achados anatomopatolológicos. Neste mesmo artigo, Waterhouse compilou mais 15 casos pediátricos ${ }^{1}$ para que, mais tarde, outros autores pudessem descrever e reconhecer essa síndrome. Dr. Carl Friderichsen foi o autor mais importante na descrição de outros casos de SWF. Embora a primeira descrição de Friderichsen date de $1917^{3}$, teve limitada repercussão por ser publicada em dinamarquês, sua língua natal. Em 1918, outra série de casos foi descrita ${ }^{4}$ pelo $\mathrm{Dr}$. Friderichsen, e em 1933 a doença começou a ser chamada de SWF. ${ }^{5}$ Em 1955, Friderichsen consolida a SWF com uma publicação de 250 casos de SWF. 6

Em homenagem ao centenário da descrição da SWF, apresentamos um caso fulminante de SWF causado por meningite meningocócica $\mathrm{C}$ vivenciado pelos médicos do Hospital Universitário da Universidade de São Paulo (HU-USP) com destaque para a condução clínico-terapêutica sob o ponto de vista das recomendações atuais de choque séptico pediátrico e contrastando com os dados históricos comparativos do relato pioneiro do Dr. Waterhouse.

\section{CASO CLÍNICO}

Paciente do sexo feminino, 3 anos e quatro meses, com história de febre de $38,50 \mathrm{C}$ há 1 dia, dor abdominal e queda do estado geral há 12 horas, sem vômitos. Evoluiu com surgimento de sufusões hemorrágicas há 4 horas da internação com início em região frontal e disseminação posterior para membros acompanhada de piora clínica. Foi admitida no pronto-atendimento do Hospital Universitário da Universidade de São Paulo (HU-USP) e imediatamente levada à sala de emergência. No exame físico de entrada encontrava-se em mal estado geral (MEG), desidratada $+/+4$, pálida, má perfusão periférica, pulsos periféricos não palpáveis, tempo de enchimento capilar (TEC) de $7 \mathrm{~s}$, petéquias difusas, taquipneica e taquicárdica, acianótica. Frequência cardíaca de200 bpm. Pressão arterial (PA) não mensurável. Peso 11 kg.

Neurologicamente torporosa, sem apresentar sinais localizatórios ou sinais meníngeos.

O exame segmentar apresentava bulhas rítmicas e normofonéticas em 2 tempos sem sopros com ausculta pulmonar normal. Abdômen normal ao exame. Antecedentes familiares: irmãos saudáveis. Tia com tuberculose. Antecedentes pessoais: vacinação em dia. Uso de sulfato ferroso por anemia.

No pronto-atendimento foi imediatamente realizada punção intra-óssea tibial bilateral e infusão rápida de volume sobre pressão $(50 \mathrm{ml} / \mathrm{kg}$ de soro fisiológico) acompanhada de intubação orotraqueal após seqüência rápida de intubação (Atropina, Fentanil, Midazolam e Rocurônio). Iniciada antibioticoterapia com ceftriaxona intravenosa e transferida para Unidade de Terapia Intensiva (UTI) Pediátrica com hipótese diagnóstica de choque séptico e meningococcemia.

Chegou à UTI-Pediátrica mantendo os sinais de choque com MEG, TEC de $6 \mathrm{~s}$, pulsos centrais cheios com pulsos periféricos não palpáveis, extremidades arroxeadas e frias e PA inaudível. Apresentava também sangramento nasal.

Após nova expansão sob pressão com soro fisiológico, colhidos os exames iniciais que mostravam acidose metabólica grave, hipoxemia, distúrbio hidroeletrolítico, insuficiência renal, pancitopenia e coagulopatia grave (Tabela 1). 
Tabela 1 - Sumário dos principais exames laboratoriais de admissão

\begin{tabular}{|c|c|c|}
\hline Bioquímica & Valores da Paciente & Valor de referência \\
\hline $\mathrm{Na}$ & 139 & $136-146 \mathrm{mEq} / \mathrm{l}$ \\
\hline K & 10 & $3,5-5,5 \mathrm{mEq} / \mathrm{l}$ \\
\hline$U$ & 71 & 10 a 50 mg/dl \\
\hline $\mathrm{Cr}$ & 1,7 & 0,4 a $1,3 \mathrm{mg} / \mathrm{dl}$ \\
\hline Glicemia & 29 & $\mathrm{mg} / \mathrm{dl}$ \\
\hline Lactato & 53,3 & arterial: 3,0 a $13,2 \mathrm{mg} / \mathrm{dl}$ \\
\hline Cálcio total & 6 & $8,6-10 \mathrm{mg} / \mathrm{dl}$ \\
\hline Cai & 0,95 & 1,15 a $1,35 \mathrm{mmol} / \mathrm{l}$ \\
\hline PCR & 93 & $<5 \mathrm{mg} / \mathrm{l}$ \\
\hline CK-MB & 147 & $<10,4 \mathrm{ng} / \mathrm{ml}$ \\
\hline Troponina I & 0,4 & $<0,4 \mathrm{ng} / \mathrm{mL}$ \\
\hline AST & 157,7 & $9-36 U / L$ \\
\hline ALT & 430 & $10-35 \mathrm{U} / \mathrm{L}$ \\
\hline Cortisol (Manhã) & 180,9 & $6-19 \mathrm{mcg} / 100 \mathrm{ml}$ \\
\hline Prot. Totais & 3,2 & $6-8 \mathrm{~g} / \mathrm{dl}$ \\
\hline Albumina & 2 & $3-5 \mathrm{~g} / \mathrm{l}$ \\
\hline Globulina & 1,2 & $1,5-3,5 \mathrm{~g} / \mathrm{dl}$ \\
\hline Gasometria arterial & Valores da Paciente & Valor de referência \\
\hline $\mathrm{pH}$ & 7,19 & $7,34-7,44$ \\
\hline $\mathrm{pO}_{2}$ & 272 & 75- $100 \mathrm{mmHg}$ \\
\hline $\mathrm{pCO}_{2}$ & 29 & $35-45 \mathrm{mmHg}$ \\
\hline $\mathrm{HCO}_{3}$ & 12 & $22-26 \mathrm{mEq} / \mathrm{l}$ \\
\hline SatO $_{2}$ & 96 & $95-98 \%$ \\
\hline Hematologia & Valores da Paciente & Valor de referência \\
\hline $\mathrm{Hb}$ & 8,8 & $10,3-15.2 \mathrm{~g} \%$ \\
\hline Leucócitos & 2800 & 4500 a $15.000 / \mathrm{mm}^{3}$ \\
\hline Plaquetas & 15.000 & 150.000 a $400.000 / \mathrm{mm}^{3}$ \\
\hline Meta /Bt /Sg /Eo /Li /Mo & $3 / 18 / 38 / 5 / 31 / 5$ & - \\
\hline
\end{tabular}


Tabela 1 - Sumário dos principais exames laboratoriais de admissão

\begin{tabular}{|c|c|c|}
\hline Coagulograma & Valores da Paciente & Valor de referência \\
\hline TP & $76 \mathrm{~s}$ & $13 \mathrm{~s}$ \\
\hline$\%$ Ativ. Plasmática & $8 \%$ & $70-100 \%$ \\
\hline INR & 12,7 & até 1,25 \\
\hline TTPA & $>180 \mathrm{~s}$ & - \\
\hline $\mathrm{R}$ & $>5,4$ & até 1,25 \\
\hline D-Dímero & $>10000$ & $<500 \mathrm{ng} / \mathrm{ml}$ \\
\hline Fibrinogênio & 135 & 175 a 400 mg/dl \\
\hline Exame Liquórico & Valores da Paciente & Valor de referência \\
\hline Aspecto & Turvo e vermelho & Límpido e incolor \\
\hline Células & 672 & $0-4 / \mathrm{mm}^{3}$ \\
\hline Hemácias & 62.976 & $0 / \mathrm{mm}^{3}$ \\
\hline Linfócitos & $6 \%$ & Predomínio de linfócitos \\
\hline Monócitos & $9 \%$ & \\
\hline Neutrófilos & $84 \%$ & \\
\hline Proteínas & 784,3 & até 40 /mm3 (lombar) \\
\hline Glicose & 285 & 2/3 da glicemia \\
\hline Contraimunoeletroforese & $(+)$ para Meningococo $\mathrm{C}$ & negativo \\
\hline
\end{tabular}

A criança persistia com hipotensão arterial que necessitando o uso de drogas vasoativas (Dobutamina, Adrenalina e Nor-Adrenalina) e hidrocortisona. Evoluiu para insuficiência renal oligúrica sendo instituída a diálise peritoneal após insucesso com uso de bomba diurética de albumina e furosemida em infusão contínua. As principais terapêuticas e monitorizações instituídas estão sumarizadas nas Figuras 1 e 2.

Expansão com $50 \mathrm{ml} / \mathrm{kg}$ de Soro fisiológico + albumina
Dobutamina
Transfusões de concentrado de hemácias e expansões adicionais com
soro fisiológico $70 \mathrm{ml} / \mathrm{kg}$
Transfusões de concentrado de hemácias, plasma e plaquetas
Adrenalina $0,3-1,5 \mathrm{mcg} / \mathrm{kg} / \mathrm{min}$ e/ou noradrenalina $0,4-4 \mathrm{mcg} / \mathrm{kg} / \mathrm{min}$,
hidrocortisona $100 \mathrm{mg} / \mathrm{m}^{2}$,terlipressina
Diálise peritoneal com catéter de Tenckoff por anúria após refratariedade
à furosemida (IV) bolus e contínuo $8 \mathrm{mg} / \mathrm{kg} /$ dia
Push de glicose para hipoglicemia (glicemia capilar= $29 \mathrm{mg} / \mathrm{dl}$ )
Insulina 0,1 Ul $/ \mathrm{kg}$ para hiperglicemia (glicemia capilar $=409 \mathrm{mg} / \mathrm{dl}$ )

Figura 1 - Principais monitorizações instituídas no controle da paciente com SWF 




Figura 2 - Principais terapêuticas instituídas no tratamento do caso com SWF

As principais recomendações do American College of Critical Care Medicine (ACCCM) para suporte hemodinâmico de choque séptico em crianças $^{7}$ e as recomendações atualizadas do Sur- viving Sepsis Campaign para o tratamento de sepse grave e choque séptico ${ }^{8}$ foram seguidas estritamente pela equipe médica. $\mathrm{O}$ cronograma de tratamento instituído foi resumido no Gráfico 1.

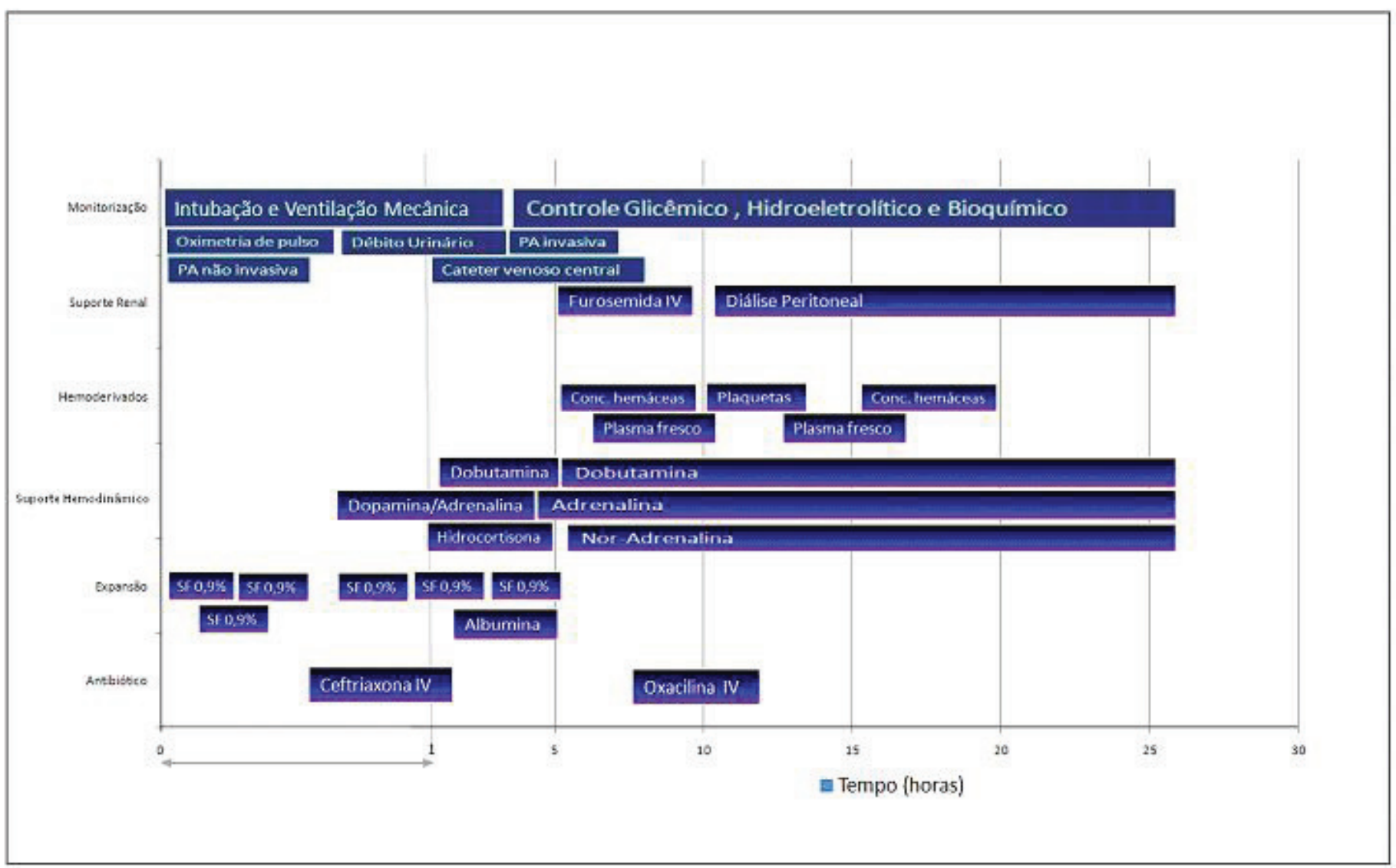

Gráfico 1 - Cronograma de terapêuticas 
Apesar da terapêutica agressiva instituída, a paciente evoluiu com choque séptico com parada cardiorrespiratória 26 horas após a entrada no pronto atendimento, sem resposta às manobras habituais de reanimação. O exame do líquido cefalorraquidiano, colhido após o óbito, confirmou meningite bacteriana por $\mathrm{N}$. meningitidis sorogrupo $\mathrm{C}$ confirmado por exame de contra-imunoeletroforese..

\section{AUTÓPSIA}

O exame macroscópico revelou quadro petequial e purpúrico compatível com purpura fulminans (PF) (Figura 3a e 3b), congestão intensa das meninges sem coleções purulentas (Figura 4), aspecto hemorrágico intenso em adrenais (Figura $5 a \mathrm{e}$ $5 b)$ e múltiplas micro- hemorragias pleurais (Figura
6) muito semelhantes aos muitos focos hemorrágicos em cápsula esplênica e renal. Havia também gastrite aguda hemorrágica em corpo e fundo gástrico.

Os principais achados microscópicos foram necrose de adrenais envolvendo mais de $70 \%$ de cada adrenal (Figura 7a e 7b), inflamação neutrofílica em leptomeninges com presença de cocos em área focal (Figura 8a e 8b), infarto renal (Figura 9) e trombos de fibrina em arteríola pulmonar (Figura 10a) relacionados ao quadro de coagulação intravascular disseminada (CIVD). Havia também raros trombos em parênquima esplênico (Figura 10b) e hiper-reatividade esplênica.

Como diagnósticos finais clínicos e anatomopatológicos foram definidos: meningite por N. meningitidis C; PF; choque séptico; CIVD e SWF.
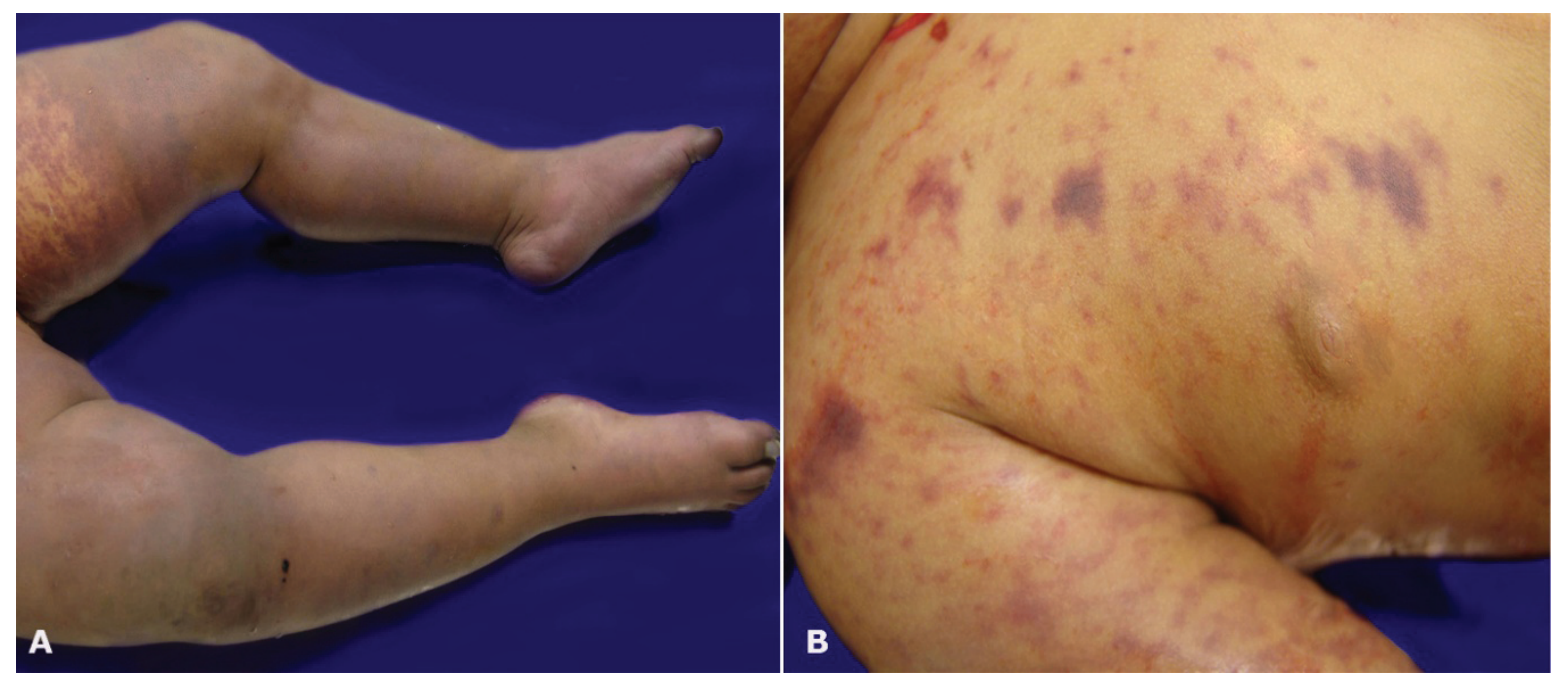

FIGURA 3- A - Membros inferiores com coloração vinhosa mais intensa nas pontas dos dedos. B - Petéquias distribuídas difusamente na pele da região torácica e dos membros superiores

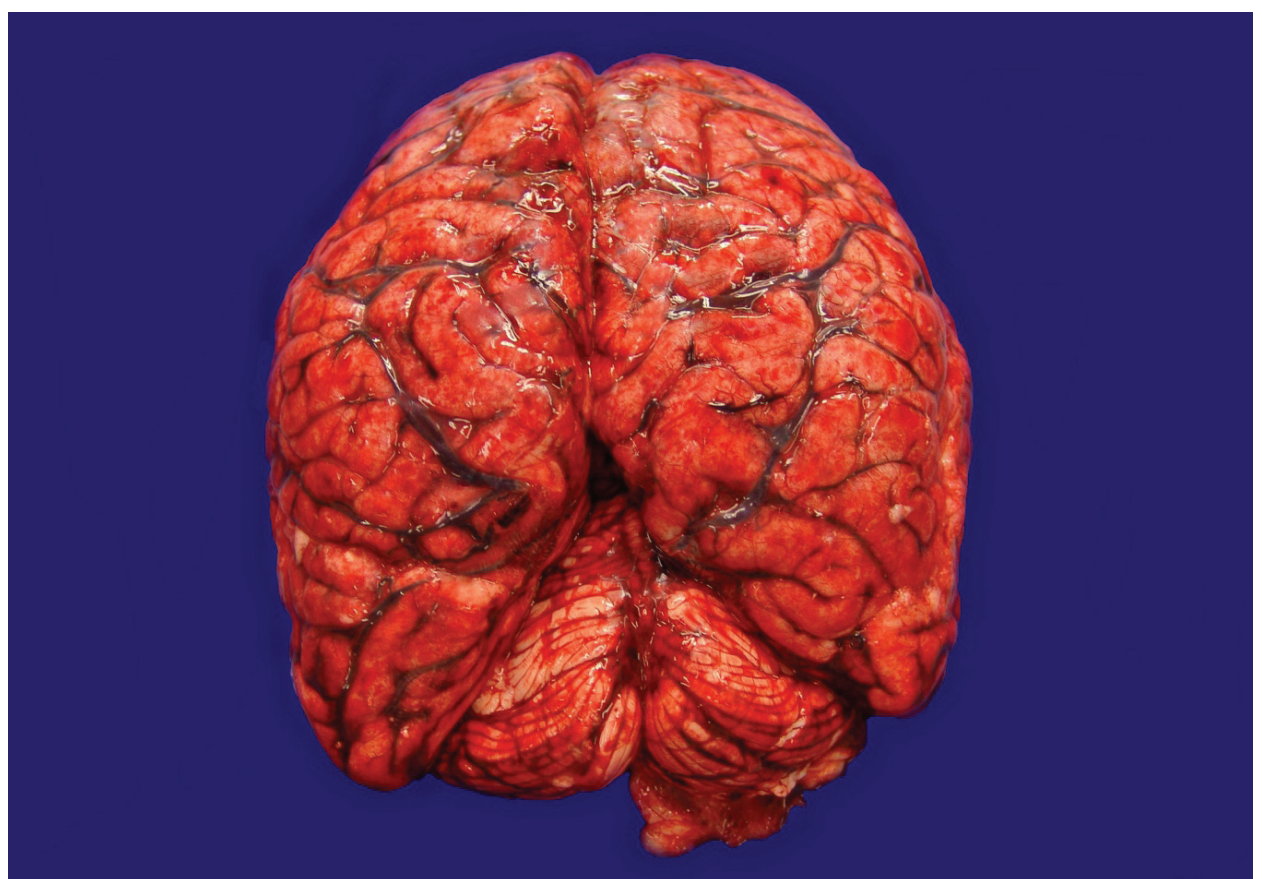

FIGURA 4 - Exame macroscópico do encéfalo mostrando congestão intensa das meninges. Não foram identificadas coleções de pus. 




FIGURA 5 -A e B - Ambas as adrenais revelavam aspecto hemorrágico intenso no exame macroscópico durante a necrópsia

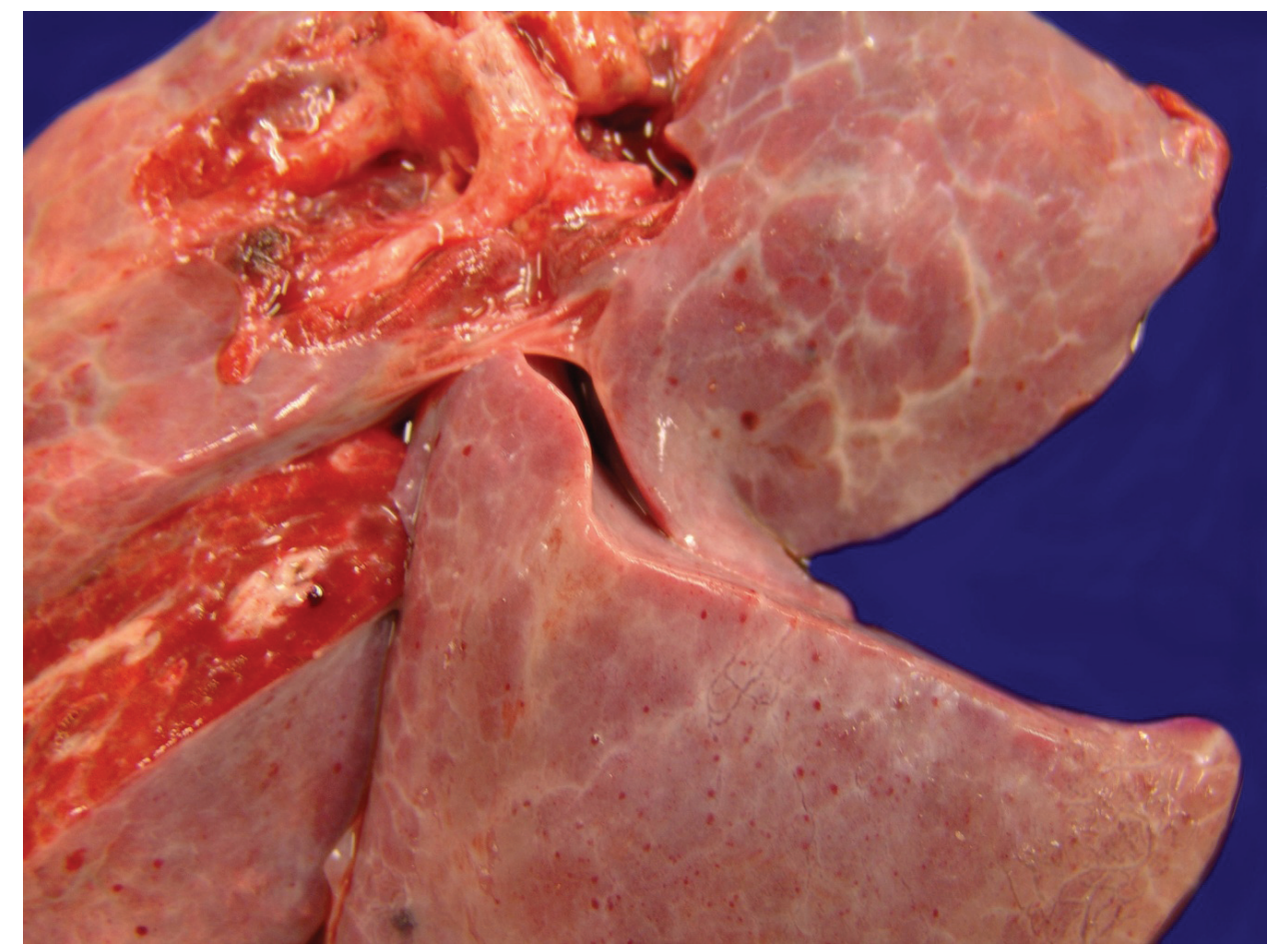

FIGURA 6 -Múltiplas microhemorragias pleurais consequentes à coagulação intravascular disseminada.



FIGURA 7 - A - Necrose hemorrágica difusa das adrenais (Fotomicrografia, HE 40x). B - Discreta preservação arquitetural, com algumas células corticais residuais em meio à necrose extensa (Fotomicrografia, HE 100x). 


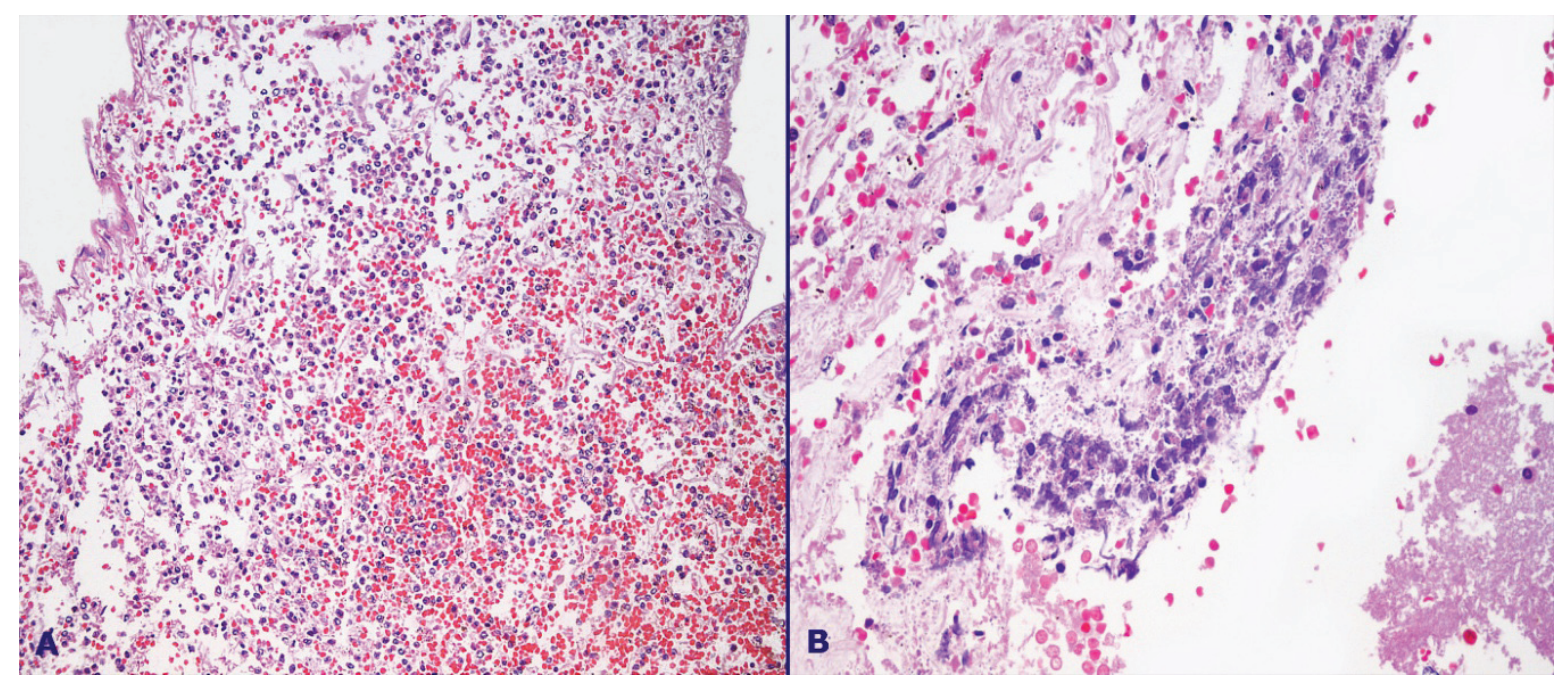

FIGURA 8 - A - Meningite piogênica aguda destacando exsudato neutrofílico intenso em leptomeninges (Fotomicrografia, HE 100x). B - Colônias bacterianas de cocos detectada focalmente (Fotomicrografia, HE 400x).

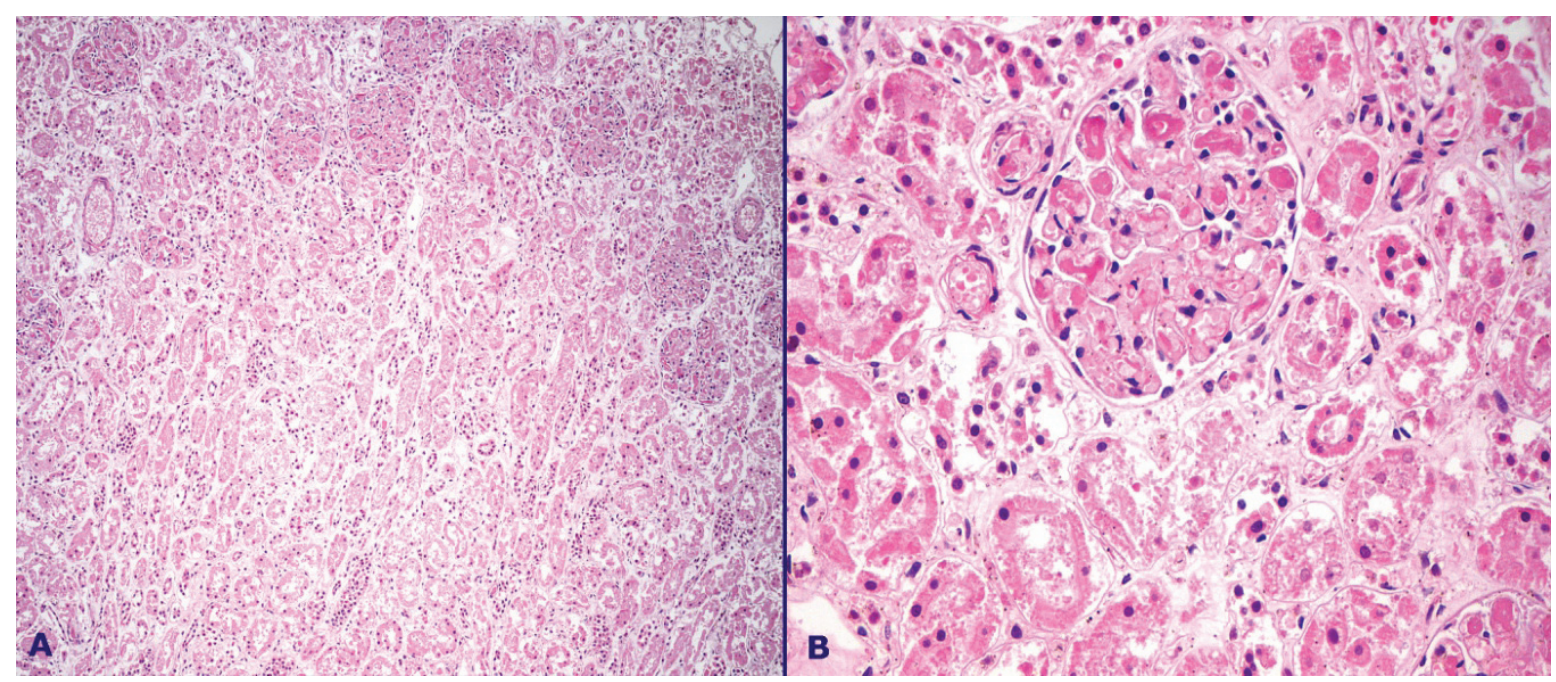

FIGURA 9 - A - Infarto renal (Fotomicrografia, HE 100x). B - Glomérulo exibindo microtrombos de fibrina na área de infarto. Havia também trombos em várias arteríolas e raras artérias arqueadas. (Fotomicrografia, HE 400x)



FIGURA 10 - A - Trombo de fibrina em arteríola pulmonar, relacionado ao quadro de CIVD (Fotomicrografia, HE 400X).

B - Raros trombos vasculares foram identificados em parênquima esplênico (Fotomicrografia, HE 400X). 


\section{DISCUSSÃO CLÍNICA}

A SWF é caracterizada por febre, colapso cardiovascular, cianose, hemorragia adrenal, lesões dermatológicas (petequiais ou purpúricas) e evolução fatal fulminante geralmente em até 24 horas do início dos primeiros sintomas. ${ }^{1,6,9} \mathrm{O}$ meningococo é o agente etiológico mais freqüente $(60-80 \%$ dos casos de SWF $)^{6,10}$, mas muitos outros agentes também foram descritos: $S$. aureus ${ }^{11}, H$. influenzae ${ }^{9}, S$. pneumoniae $^{12,13}$, estreptococo beta hemolítico ${ }^{12}$, Morganella morganii ${ }^{14}$, Enterococcus faecium ${ }^{14}$, Klebsiella ${ }^{14}$, Pasturella ${ }^{14}$, Capnocytophaga canimorsus, Micobactérias e vírus da varicela. A asplenia pode colaborar com o desenvolvimento de SWF ${ }^{13}$ por favorecer infecções bacterianas.

Meningococcemia refere-se à sepse purpúrica fulminante do meningococo. O nome SWF deve ser reservado para a presença de septicemia fulminante em que se comprove destruição hemorrágica das adrenais. ${ }^{15}$ Como o agente causal nem sempre é o meningococo $0^{9,11-14}$, o termo sepse fulminante purpúrica proposto em $1953^{15}$ ou púrpura febril séptica ${ }^{16}$ são preferencialmente sugeridos ao invés de meningococcemia quando ainda não se tem definitivamente os resultados microbiológicos.

O comprometimento agudo das glândulas adrenais explica a maioria dos sinais e sintomas clínicos exuberantes apresentados por esta paciente. A manifestação predominante de crise adrenal é o choque, mas os pacientes geralmente apresentam sintomas inespecíficos como anorexia, náusea, vômito, dor abdominal, fraqueza, fadiga, letargia, febre, confusão ou coma. ${ }^{17}$

A faixa etária pediátrica é sempre a mais acometida, particularmente crianças menores de 2 anos que respondem por $70 \%$ dos casos. ${ }^{18,19} \mathrm{Em}$ 1944 , dos 150 casos descritos até a época, houve 19 ocorrências $(12,7 \%)$ em adultos e $87,3 \%$ em crianças. $^{19}$

A constatação da presença de $P F$ em nossa paciente colabora com o diagnóstico da SWF, mas sua presença não é obrigatória, pois há relatos de ausência de hemorragia adrenal em $26 \%$ dos casos fatais de PF. ${ }^{20}$ Ademais, na compilação dos casos feita pelo Dr. Waterhouse, $80 \%$ dos casos de apoplexia suprarenal tinham $P F^{1}$. $P F$ é descrita desde $1884^{21}$ pelo quadro purpúrico confluente associado à intensa coagulação intravascular disseminada, com desordem trombótica que pode levar à necrose de pele e/ou amputação de membros. ${ }^{22}$ No nosso relato, fica evidente a presença de $P F$ pelo quadro dermatológico característico e presença de CIVD clínica e laboratorial.

A prioridade no manejo do choque séptico é sempre o tratamento otimizado imediato evitando a deterioração das condições clínicas do paciente. Neste sentido, a prioridade foi a manutenção de vias aéreas, o suporte ventilatório, o restabelecimento rápido das condições hemodinâmicas com o uso de expansores do volume extracelular, drogas vasoativas e hemoderivados, o tratamento antimicrobiano na primeira hora do choque e todas as outras recomendações da $\mathrm{ACCCM}^{7}$ e Sepsis Survival Campgnain. ${ }^{8}$ A SWF é invariavelmente fatal se a terapêutica com glicocorticóide não é iniciada precocemente. ${ }^{19,23}$ Entretanto, a instituição de diagnóstico e tratamento precoces pode ser muito difícil quando os sintomas não são específicos. No fluxograma de tratamento recomendado pela $\mathrm{ACCCM}$, a terapia com corticoesteróide deve ser instituída na suspeita de insuficiência adrenal ou de $\mathrm{SWF}^{7}$, o que correspondeu ao uso precoce de hidrocortisona $100 \mathrm{mg} / \mathrm{m}^{2}$ no primeiro dia e $50 \mathrm{mg} / \mathrm{m}^{2}$ a partir de então. Interessante notar que o nível sérico de cortisol colhido era de $100 \mathrm{mcg} /$ dl, o que mostrava que a paciente não apresentava em insuficiência adrenal absoluta. Pizarro e colaboradores $^{24}$ relataram, após realização de teste com corticotropina, insuficiência adrenal relativa em $26 \%$ dos casos de choque séptico dos quais $80 \%$ com choque resistente a catecolaminas.

A coleta do líquor não é prioridade inicial ainda que a suspeita inicial seja meningite bacteriana. As medidas de isolamento, quimioprofilaxia para contactantes e uso de antibiótico de amplo espectro parenteral para o sistema nervoso central não devem ser retardadas. A insistência nesta coleta à entrada pode levar ao risco desnecessário de colapso cardiorrespiratório durante o procedimento e atraso de medidas restauradoras vitais. Após estabilização da paciente, os exames de cultura ou teste de antígenos permitirão documentar o agente causal num momento mais oportuno. Houve o isolamento tardio de Meningococo $\mathrm{C}$ nesta paciente, mas todas as condutas necessárias para o tratamento contra este agente já tinham sido adiantadas.

O tratamento dos casos deve ser agressivo com inclusão precoce de antibióticos de amplo espectro e terapêutica com corticoticoesteróides após o suporte inicial com ressuscitação volumétrica e cardiovascular.

Apesar de se discutir o uso de hemoderivados (plasma fresco congelado e concentrado de plaquetas), proteína $\mathrm{C}$ ativada, plasmaférese e anticoagu- 
lação (heparina, antitrombina III, rtPA) como opções de tratamento como para $P F^{22,25}$, verifica-se que no tratamento atual da SWF nenhuma terapêutica tem apresentado resultado convincente que altere a taxa de mortalidade. A prevenção com vacinação dos pacientes contra o meningococo, o agente causal mais importante, tem sido uma estratégia para minimizar a SWF, mas deve-se lembrar sempre dos outros agentes para os quais não há vacinas disponíveis.

Convém lembrar que na época pré-antibioticoterapia, em 1911, Dr. Waterhouse chegou a coletar culturas das adrenais, baço e do líquor que resultaram negativas, o que mostrava a preocupação do Dr. Waterhouse sobre uma possível causa infecciosa da SWF. O relato pioneiro da SWF pelo Dr. Waterhouse serve também como um documentário de época e permite ver a evolução da Medicina neste último século. Neste relato de $1911^{1}$, Dr. Waterhouse usou tudo o que the era disponível: mediu a temperatura (termômetro clínico descoberto em 1866, Allbutt) e fez a microscopia ótica (presente desde o século XVII). Não descreveu a pressão arterial no relato, pois a medida de pressão arterial não invasiva era razoavelmente nova (1905, Nikolai Korotkoff) ${ }^{26}$ e Dr. Waterhouse pode não ter tido acesso à mesma, considerando paciente pediátrico. Dr. Waterhouse não relatou nenhum tratamento instituído ao paciente de 8 anos sob seus cuidados, mas como culpá-lo, se a descoberta da penicilina por Alexander Flemming aconteceria somente em 1928? Vale lembrar que Dr. Waterhouse falava de apoplexia suprarrenal em 1911 e o isolamento da substância cortisona ocorrereu somente em 1935 (Reichstein-Kendall-Heinch) e rendeu aos autores em 1951, o prêmio Nobel. Entender a primeira descrição da SWF é entender um pouco da evolução da história

\section{REFERÊNCIAS}

1. Waterhouse R. A case of suprarenal apoplexy. Lancet. 1911;177(4566):577-8.

2. Varon J, Chen K, Stembach GL. Rupert Waterhouse and Carl Friderichsen: adrenal apoplexy. J Emerg Med. 1998;16(4):643-7.

3. Friderichsen C. Binyreaopoleski hos Smaaborn. Ugeskrift for Laeger. 1917;79:1817-26.

4. Friderichsen C. Nebennierenapoplexie bei kleinen Kindern [adrenal apoplexy in small children]. Jahrbuch fur Kinderheilkunde. 1918;87:109-25

5. Bamatter F. Fulminate Meningokokkensepsis. Zur Atiologie des Syndroms von Waterhouse-Friderichsen. Jahrbuch fur Kinderheilkunde. 1934;142:129-62. da medicina atual, com suas descobertas ainda um tanto claras em nossa memória.

\section{CONCLUSÃO}

Cem anos em medicina representam um imenso progresso tecnológico. Apesar dos inúmeros avanços alcançados pela medicina desde 1911, a evolução fatal da SWF foi compartilhada por ambos os pacientes pediátricos com um século de diferença. Apesar do melhor entendimento clínico, microbiológico, histopatológico e laboratorial da SWF na atualidade e, a despeito de todo o arsenal terapêutico disponível e da condução terapêutica sistematizada, infelizmente ainda não é possível garantir a sobrevida dos pacientes pediátricos com SWF. Certamente, a SWF continuará a ser um desafio para o futuro que conclamará a necessidade de novas descobertas científicas na medicina, visando combater eficazmente a síndrome infecto-contagiosa mais temida em pronto atendimento pediátrico.

\section{AGRADECIMENTOS}

Agradecemos a todos os componentes das equipes de médicos, residentes, enfermeiros, fisioterapeutas, farmacêuticos, assistentes sociais e funcionários do serviço de anatomia patológica do Hospital Universitário da USP que colaboram de forma direta e anônima com o atendimento otimizado de todos os casos de choque séptico que rotineiramente convergem ao Hospital Universitário. Agradecemos especialmente pela colaboração de Rosa Maria da Conceição Zanardi na produção visual das imagens macroscópicas e microscópicas deste caso.

6. Friderichsen C. Waterhouse-Friderichsen syndrome. Acta Endocronologica.1955;18(4):482-92.

7. Brierley J, Carcillo JA, Choong K, Cornell T, Decaen A, Deymann $A$, et al. Clinical practice parameters for hemodynamic support of pediatric and neonatal septic shock: 2007 update from the American College of Critical Care Medicine. Crit Care Med. 2009;37(2):666-88.

8. Dellinger RP, Levy MM, Carlet JM, Bion J, Parker MM, Jaeschke $R$ et al. Surviving Sepsis Campaign: international guidelines for management of severe sepsis and septic shock: 2008. Crit Care Med. 2008;36(4):1394-6.

9. Beach RC, Clayden GS, Eykyn SJ. Waterhouse-Friderichsen syndrome caused by Haemophilus influenzae type b. Br Med J. 1979;2(6198):1111.

10. Monfort JA, Mehrlingm J. The Waterhouse-Friderichsen Syndrome. Review of the Literature and report of a case with autopsy. Am J Dis Child. 1941;62(1):144-9. 
11. Adem PV, Montgomery CP, Husain AN, Koogler TK, Arangelovich V, Humilier M, et al. Staphylococcus aureus sepsis and the Waterhouse-Friderichsen syndrome in children. N Engl J Med. 2005;353(12):1245-51.

12. J Hamilton D, Harris MD, Foweraker J, Gresham GA. Waterhouse-Friderichsen syndrome as a result of nonmeningococcal infection. Clin Pathol. 2004;57(2):208-9.

13. Vincentelli C, Molina EG, Robinson MJ. Fatal pneumococcal Waterhouse-Friderichsen syndrome in a vaccinated adult with congenital asplenia. Am J Emerg Med. 2009 ;27(6):751.e3-5.

14. Tourrel F, Gouin P, Dureuil B, Veber B. Waterhouse-Friderichsen syndrome associated to a Morganella morganii and Enterococcus faecium peritonitis. Ann Fr Anesth Reanim. $2007 ; 26(10): 869-72$.

15. BjöRKLUND SI. The Waterhouse-Friderichsen Syndrome. Acta Paediatrica1953; 42(1):77-83.

16. Hsin SH, Fontes MAS, Bousso A, Fernandes ICOF, Cordeiro AMG, Miyake RS, Fernandes JC, Ejzenberg B, Okay Y. Therapeutic of septic children with purpuric presentation with two antibiotic schedules. J Pediatr 1988;74(4):315-24.

17. Nieman LK, Lacroix A, Martin KA. Clinical manifestations of adrenal insufficiency in adults. UpToDate.(last updated: Sept 29, 2009).

18. KWEDAR AT. The Waterhouse-Friderichsen Syndrome: report of a case in an adult. Ann Intern Med. 1942;16(4):787-91.
19. Newman AA. The Waterhouse-Friderichsen Syndrome: Report of three cases in adults with necropsy findings. Yale J Biol Med. 1945;18(1):31-36.

20. Leclerc F, Delepoulle F, Martinot A, Diependaele JF, Houque D, Hue V.[Frequency of adrenal hemorrhage in fatal forms of purpura fulminans in children. Etiopathogenic and therapeutic considerations]. Pediatrie. 1988;43(6):545-50.

21. Urbaniak JR, O'Neil MT, Meyer LC. Purpura fulminans. J Bone Joint Surg Am. 1973;55(1):69-77.

22. Chalmers E, Cooper P, Forman K, Grimley C, Khair K, Minford A, Morgan M, Mumford A. Purpura fulminans: recognition, diagnosis and management. Arch Dis Child. 2011 Jan 12. [Epub ahead of print]

23. Kovacs KA, Lam YM, Pater JL. Bilateral massive adrenal hemorrhage. Assessment of putative risk factors by the case-control method. Medicine (Baltimore). 2001;80(1):45-53.

24. Pizarro, CF; Troster EJ; Damiani D; Carcillo JA. Absolute and relative adrenal insufficiency in children with septic shock. Crit Care Med. 33(4):855-9.

25. Nolan J, Sinclair R. Review of management of purpura fulminans and two case reports. $\mathrm{Br} J$ Anaesth 2001;86(4):581-6.

26. Shevchenko YL, Tsitlik JE. 90th Anniversary of the Development by Nikolai S. Korotkoff of the Auscultatory Method of Measuring Blood Pressure. Circulation. 1996;94(2):116-8.

\section{Conflito de Interesse: Não}

Submetido em: 03 de Março de 2011

Aceito em: 11 de Março de 2011

Correspondência: Divisão de Clínica Pediátrica - Unidade de Terapia Intensiva

Av. Prof. Lineu Prestes, 2565 - Cidade Universitária - São Paulo - SP - Brasil

CEP: 05508-000 - Tel.: +55.11. 3091.9248

E-mail: shieh@hu.usp.br 